\title{
Reproducing Standard SCME Channel Models for Massive MIMO Base Station Radiated Testing
}

\author{
Wei Fan, Fengchun Zhang, Tommi Jämsä, Mattias Gustafsson, Pekka Kyösti and Gert F. Pedersen
}

\begin{abstract}
Massive MIMO is a multi-user technology, where radio base stations (BSs) are equipped with a large number of antennas to simultaneously serve many terminals in the same time-frequency resource. Performance evaluation of such largescale antenna systems in the design and development stage is challenging. In this paper, we propose to evaluate massive MIMO BSs with a sectorized multi-probe anechoic chamber (MPAC) setup. A sectorized MPAC setup with 16 probe antennas distributed uniformly within $\left[-60^{\circ}, 60^{\circ}\right]$ in azimuth domain is utilized to reproduce target channel models. A $8 \times 8$ and a $16 \times 16$ uniform planar array at $3.5 \mathrm{GHz}$ are selected as the BS under evaluation, respectively. Radio channel emulation accuracies in terms of power-angular spectrum, spatial correlation and beamforming pattern are investigated for the proposed MPAC setup and desired channel models.
\end{abstract}

Index Terms-Anechoic chamber, massive MIMO base stations, over-the-air evaluation, radio channel emulation

\section{INTRODUCTION}

Massive multiple-input multiple-output (MIMO) techniques have arisen as enabling technologies to deal with the high data and reliable communication requirement in $5 \mathrm{G}$ cellular systems [1]. Massive MIMO is a multi-user technology, where radio base stations (BSs) are equipped with a large number of antennas to simultaneously serve many terminals in the same time-frequency resource. The promising features of utilizing excessive number of antenna elements at the BS side have been discussed extensively from a theoretic point of view in many works in the literture, see e.g. [1], [2]. Meanwhile, there has been a strong ongoing effort to design practical massive MIMO systems both from academia and industry, see e.g. promising results reported in [1], [3].

Radio performance evaluation is required in different phases of product development from early research prototypes, design optimization, to actual product approval for roll-out. Conventional BSs are often evaluated by connecting coaxial cables to the BS antenna ports, i.e. with antennas bypassed [4]. However, this becomes problematic for massive MIMO, where hundreds of cable connections to the antenna ports and respective hardware are expected. In addition, antenna ports might be unaccessible for BSs in future $5 \mathrm{G}$ systems. Thus, there is a strong need to replace conductive testing.

Historically, over-the-air (OTA) testing has been used to evaluate radio performance of handset antenna systems [5][7]. The testing is done in a wireless manner (i.e. over-the-air),

Wei Fan, Fengchun Zhang and Gert F. Pedersen are with the Antennas, Propagation and Radio Networking section at the Department of Electronic Systems, Aalborg University, Denmark (email: wfa@es.aau.dk).

Tommi Jämsä and Mattias Gustafsson are with Huawei Technologies Sweden AB, Gothenburg, Sweden.

Pekka Kyösti is with Keysight Technologies and Oulu University, Finland without a need to break the device. Various OTA methodologies have been proposed to evaluate UE systems with multiple antennas, where the multi-probe anechoic chamber (MPAC) method has been selected in the standardization for UE systems with multiple antennas [6]. The MPAC method is attractive, since realistic propagation environments can be physically synthesized and controlled in a shielded anechoic chamber, via controlling the signals radiated from multiple probe antennas. Some works have discussed the applicability of MPAC method for massive MIMO BS performance evaluation. In [8], [9], possible testing systems are discussed for BS performance evaluation. In [10], suitable measurement distances and physical dimensions of the MPAC setups for the performance evaluation of massive MIMO BSs in anechoic chambers are investigated. Standardization work on radiated conformance testing of BSs, equipped with active antenna systems, is ongoing [7].

In this paper, we first discussed OTA testing for massive MIMO BSs in sectorized MPAC setups in Section II. After that, we demonstrate the simulation results for reproducing standard SCME channel models for massive MIMO BS testing in a sectorized setup in Section III and Section IV. Section V concludes the paper.

\section{MASSIVE MiMO OTA TESTING IN MPAC SETUPS}

An illustration of typical cellular propagation environment is shown in Figure 1. For the UE side, since the scatterers are often nearby and located around the UE, the angle profiles are less specular. For example, a root mean square (RMS) angle spreads (ASA) of $35^{\circ}$ are specified for each cluster for the SCME UMa and SCME UMi channel models at the UE side, as shown in Figure 2. Further, multipath components can impinge on the UE from arbitrary directions. Note that the UMa and UMi channel models are essentially the same SCME models, but with different channel parameters.

An illustration of the typical MPAC configuration for UE testing is shown in Figure 3, where the system consists of a BS emulator, one or several channel emulator and multiple probe antennas. The UE is often located in the center of the OTA ring. For UE OTA testing, a uniform configuration of the OTA probes over the azimuth plane is often adopted [12], since it offers the possibility to recreate any spatial channel model without relocation of the probe antennas.

A sectorized MPAC setup would be more appropriate for massive MIMO BS testing, as shown in Figure 4:

- From point of view of propagation environments. The angle profiles at the BS side have small angle spread due 


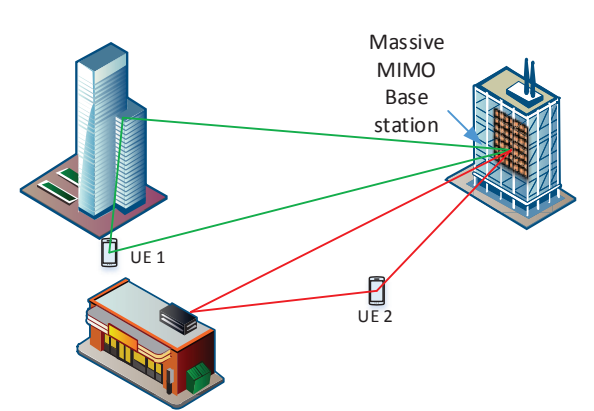

Figure 1. Real multipath environments for cellular systems.
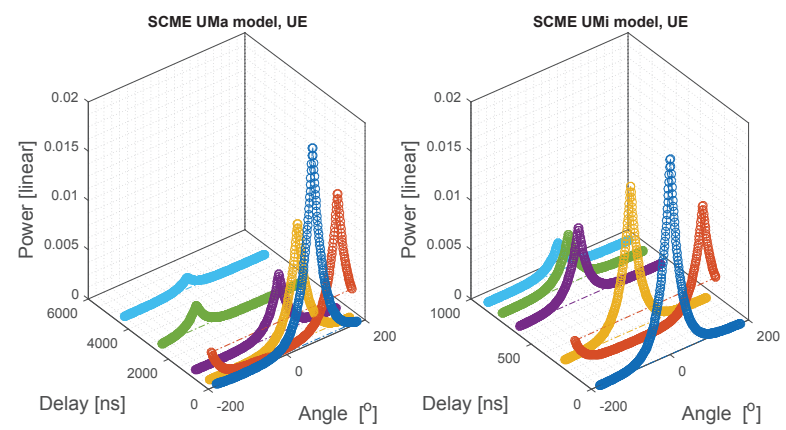

Figure 2. Power-angle-delay profiles for the SCME UMa channel model (left) and SCME UMi channel model (right) at the UE side.

to the fact that the BS is placed higher and further away from scatterers. Furthermore, paths might be blocked due to practical installations and therefore field of view of the array might be limited, see e.g. Figure 1 .

- From point of view of BS antenna array. The target coverage area of a BS antenna is smaller than that of a UE antenna. While a UE antenna by practical reasons needs to receive and transmit from/to all angles, a BS antenna normally is restricted to receive and transmit power in a desired angular zone. This angular zone normally is about $60^{\circ}$ in azimuth and about $20^{\circ}$ in elevation.

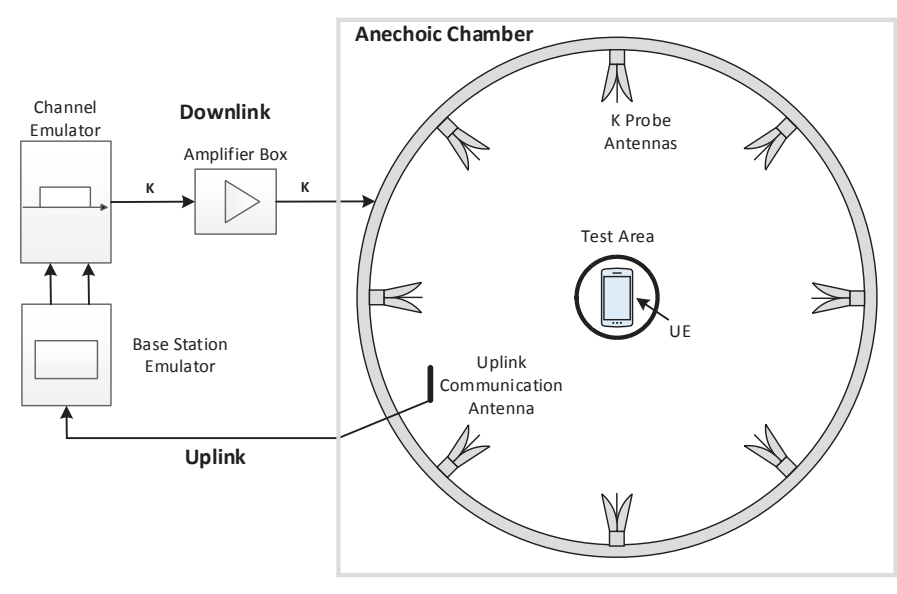

Figure 3. An illustration of the MPAC setup for UE performance evaluation $K$ OTA antennas are connected to $K$ radio frequency (RF) interface channels.

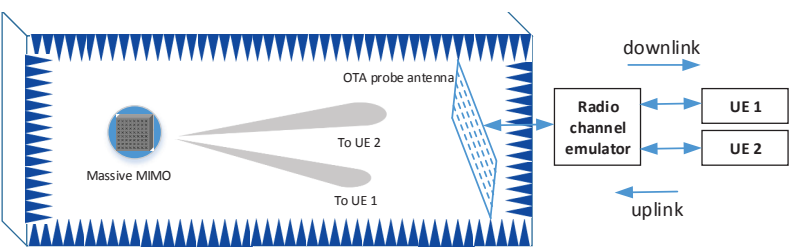

Figure 4. Sectorized MPAC setups for radiated testing of massive MIMO BS

- From point of view of setup cost. A sectorized MPAC configuration has the potential to reduce the system cost. Firstly, a sectorized probe configuration has the potential to save cost, via reducing the required number of fading channel emulators and respective hardware resource. Secondly, the DUT can be placed further away from OTA antennas to utilize the full chamber dimension.

An illustration of the proposed sectorized MPAC setup is shown in Fig. 4. For massive MIMO BS testing, BS and UE positions are swapped compared to Figure 3, which requires a larger test area. UEs or UE emulators are used to emulate the user ends of the radio link. Multiple UEs might be needed for performance testing. Radio channel emulators and OTA antennas are utilized to mimic spatial channels for the desired user and interfering users.

\section{Channel EMUlation In SECTORIZED MPAC SETUPS}

\section{A. Target channel models}

In this paper, two representative $2 \mathrm{D}$ channel models, i.e. SCME UMa and SCME UMi [11], are selected for the sake of simplicity. The channel parameters, e.g. delay, power, angle of departure (AoD), angle spread of departure (ASD), angle of arrival (AoA), angle spread of arrival (ASA) for each cluster can be found in [11]. Note that both channel models are assumed to be vertically polarized for discussion for simplicity.

The mean AoDs of the composite power angle spectra at the BS side can be calculated as in (1)

$$
\bar{\theta}=\frac{\sum p_{i} \theta_{i}}{\sum p_{i}}
$$

where $p_{i}$ and $\theta_{i}$ denote the power and azimuth angle of the $i$-th cluster, respectively. In the SCME UMa and SCME UMi channel models, the mean AoD of the composite power angle spectra at the BS side is $\bar{\theta}=84.3^{\circ}$ and $\bar{\theta}=21.7^{\circ}$, respectively. In this paper, the mean AoDs of both channel models are shifted to $0^{\circ}$ for simplicity.

The power-angle-delay profiles for the modified SCME UMa channel model and SCME UMi channel model at the BS side are shown in Figure 5. As we can see, each path is quite specular, with cluster RMS angle spread $2^{\circ}$ for the UMa and with $5^{\circ}$ for the UMi case, respectively. Further, the spatial profiles of both channel models are spatially confined, within $\left[-60^{\circ}, 60^{\circ}\right]$ angle region, as shown in Figure 5.

\section{B. Sectorized MPAC configuration and DUT}

In this paper, a 2D sectorized configuration is utilized to reproduce the $2 \mathrm{D}$ SCME channel models. Note that a 

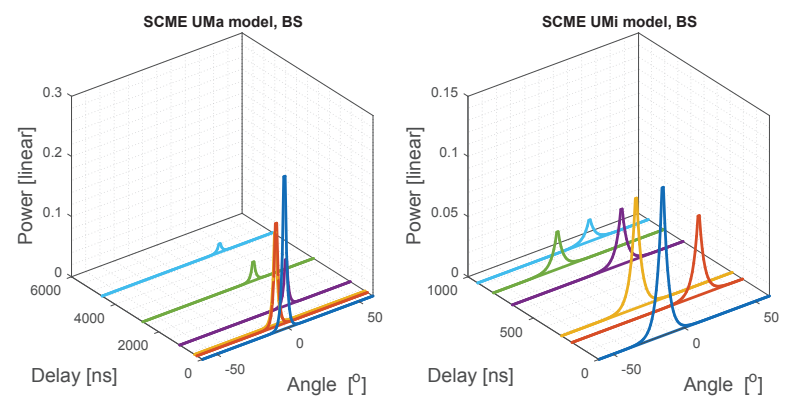

Figure 5. Power-angle-delay profiles for the modified SCME UMa channel model (left) and SCME UMi channel model (right) at the BS side. Note that the angle axis is limited to $\left[-60^{\circ}, 60^{\circ}\right]$.

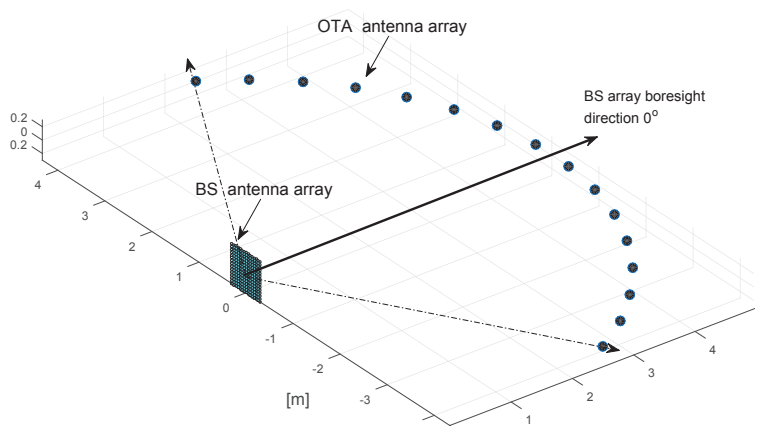

Figure 6. An illustration of the probe configuration.

$3 \mathrm{D}$ configuration is required to reproduce $3 \mathrm{D}$ channel models, i.e. with elevation domain, see e.g. discussions in [10]. $K=16$ probe antennas uniformly distributed in azimuth angle $\left[-60^{\circ}, 60^{\circ}\right]$ are utilized on a circular probe configuration with $R=5 \mathrm{~m}$. 16 OTA probe antennas are selected as an example in the simulation, since this configuration can be supported by a single radio channel emulator (e.g. Propsim F32). The antenna array boresight direction is defined to be $0^{\circ}$. The carrier frequency is set to be $3.5 \mathrm{GHz}$, as it is widely used for massive MIMO analysis in the literature. Isotropic antenna patterns are assumed for the OTA probes and mutual coupling is not considered for the sake of simplicity. Note that the sectorized MPAC configuration can only support modeling channel models whose spatial profiles are confined within the sectorized angle region. To have more flexibility, a more uniform MPAC configuration might be needed. Two BS antenna arrays are considered as DUT, i.e. one $8 \times 8$ uniform planar array (i.e. 64 antenna elements) and one $16 \times 16$ (i.e. 256 antenna elements) uniform planar array with half wavelength element spacing, as illustrated in Figure 7. The physical size of the antenna arrays are around $0.3 m \times 0.3 m$ and $0.64 m \times 0.64 m$, respectively. Isotropic antenna patterns for antenna elements are used in the simulations.
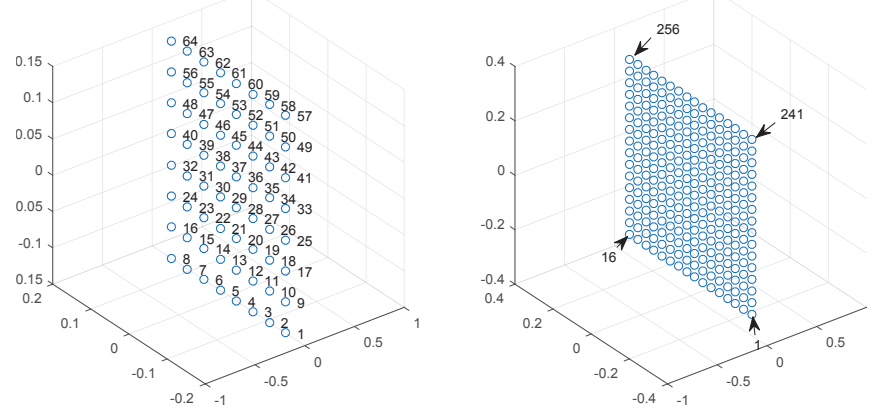

Figure 7. An illustration of the massive MIMO BSs in the simulations: $8 \times 8$ uniform planar array (left) and $16 \times 16$ uniform planar array (right).
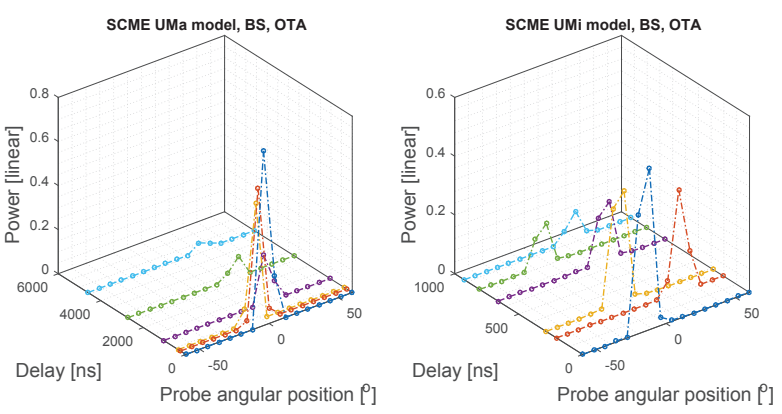

Figure 8. Power-angle-delay profiles for the SCME UMa channel model (left) and SCME UMi channel model (right) at the BS side. The ASA of the cluster at the $\mathrm{BS}$ is $2^{\circ}$ for the $\mathrm{UMa}$ and $5^{\circ}$ for the UMi, respectively. Note that the angle axis is limited to $\left[-60^{\circ}, 60^{\circ}\right]$.

\section{Channel emulation technique}

The PFS technique has gained its popularity in the industry due to its capability to emulate all dimensions of the standardized channel models [13], with only power calibration required for OTA antennas. With the PFS technique, each cluster is emulated by several probe antennas. For each cluster, the BS side spatial characteristics are reconstructed by allocating appropriate power weights to the fading signals from the probes. To obtain the optimal power weights allocated to the probe antennas, the objective is to minimize the deviation between target spatial correlation and emulated spatial correlation [13]. The obtained power weights for the target channel models are shown in Figure 8. As we can see, the emulate power angle delay profiles match target profiles well. The deviation is due to limited OTA antennas.

\section{Channel emulation accuracy With MPAC SETUPS}

\section{A. Spatial correlation}

The correlation between signals received on the antenna elements can be calculated according to the correlation definition. The correlations between signals received by the first antenna and the other antennas under target and emulated channel models for both channel models for the $8 \times 8$ uniform planar array is shown in Figure 9. As we can see, good agreement 


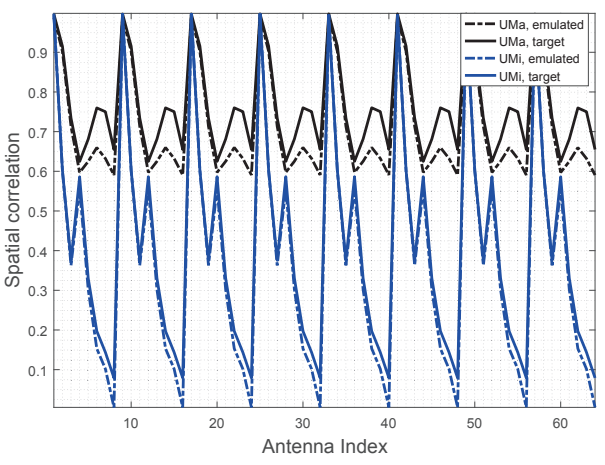

Figure 9. Target and emulated spatial correlation between 1 st antenna element and other antenna elements on the $8 \times 8$ uniform planar array, as illustrated in Figure 7

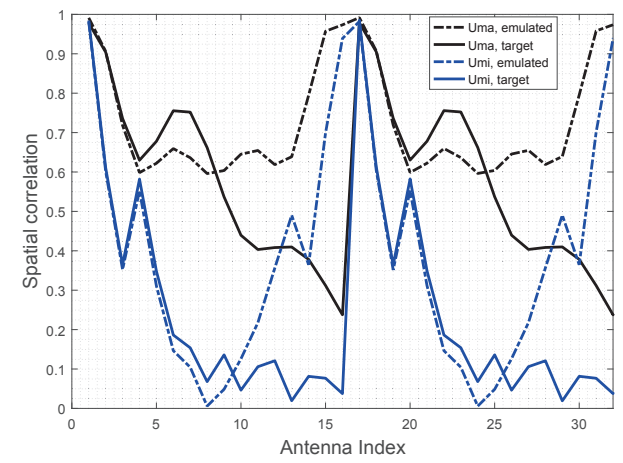

Figure 10. Target and emulated spatial correlation between 1st antenna element and other antenna elements on the $16 \times 16$ uniform planar array, as illustrated in Figure 7. Note that only antenna elements in two bottom columns are shown.

between the spatial correlations calculated under target channel models and under emulated channels can be achieved for both channel models. The agreement is better for the SCME UMi channel models due to the larger cluster angle spread in the target channel. For the SCME UMa model, a deviation up to 0.1 exists in the high correlation region, which might slightly impact the system performance. Note that the periodic pattern in spatial correlation curves are due to the fact that the target and emulated channels are concentrated on a $2 \mathrm{D}$ azimuth plane. Therefore, we expect same correlation values for antenna elements on different elevation columns.

The correlation values for target and emulated channel models for the $16 \times 16$ uniform planar array are shown in Figure 10. We observe good agreement for both SCME UMa and UMi channel models up to around $5 \lambda$ (i.e. antenna separation between element 1 and element 11). Large deviation exists in spatial correlation accuracy for antenna separation above $5 \lambda$. The deviation is due to that fact that a larger test object requires more probe antennas. Note that the deviation due to limited physical dimension of the MPAC (i.e. $R=5 \mathrm{~m}$ ) is negligible in the simulation.

\section{B. Beamforming power pattern}

The beamforming power patterns of the planar arrays under the target and emulated channel models for both channel

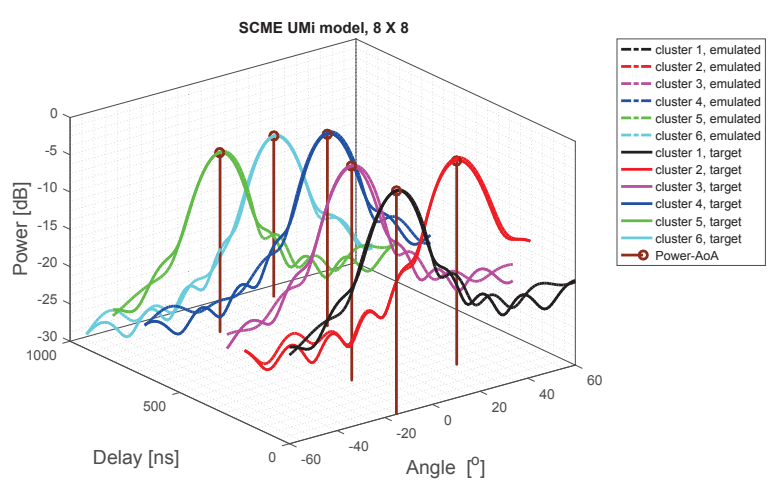

Figure 11. Beamforming power pattern of the target and emulated SCME UMi channel models with the $8 \times 8$ planar array.

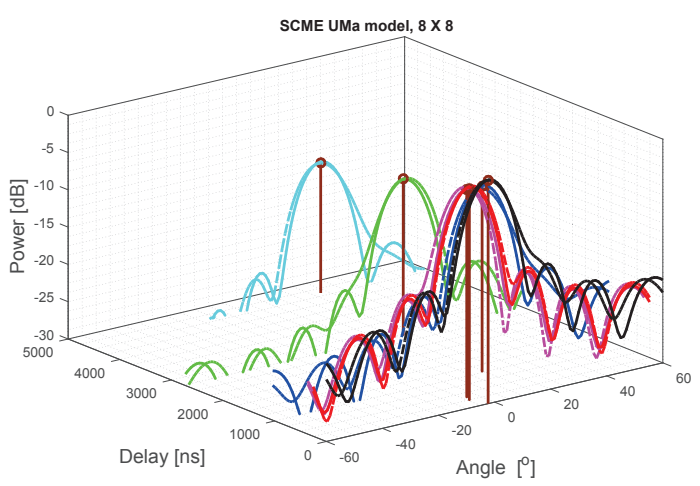

Figure 12. Beamforming power pattern of the target and emulated SCME UMa channel models with the $8 \times 8$ planar array.

models are shown in Figure 11 (SCME UMi channel model and $8 \times 8$ planar array), Figure 12 (SCME UMa channel model and $8 \times 8$ planar array), Figure 13 (SCME UMi channel model and $16 \times 16$ planar array) and Figure 14 (SCME UMa channel model and $16 \times 16$ planar array), respectively. As we can, good match in terms of the main beam, sidelobes and beam shape can be achieved for the SCME UMi channel model with $8 \times 8$ planar array. As for the SCME UMa channel model, though we can still achieve good agreement for the main beam and beam shape, some deviations exist in the sidelobes. This is introduced by the small deviations in spatial correlation emulation accuracy, as discussed earlier in Figure 9. Note that the mean AoD of each target cluster is plotted in the figure as a reference for visualization purpose.

As for the $16 \times 16$ planar array cases, though good agreement can be observed for the beam shape, as shown in Figure 13 and Figure 14, some deviations exist in the main beam and sidelobes. This is due to the fact that the spatial resolution of the planar array is higher than the probe angular separations. This effect is clearly observed in Figure 15, where a peak around target angle-of-arrival exists in the beamforming power pattern under target channel models, while two peaks exist at the two dominant probe directions for the beamforming pattern under emulated channel models. 


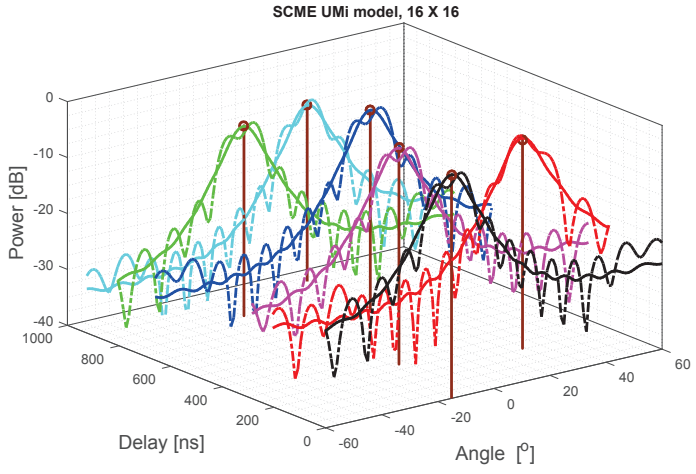

Figure 13. Beamforming power pattern of the target and emulated SCME UMi channel models with the $16 \times 16$ planar array.

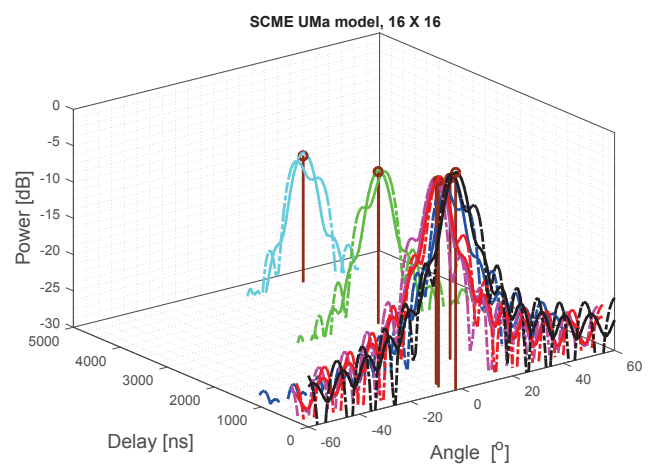

Figure 14. Beamforming power pattern of the target and emulated SCME UMa channel models with the $16 \times 16$ planar array.

\section{CONCLUSION}

This paper aims to reproduce SCME channel models with a sectorized MPAC configuration for massive MIMO BS performance evaluation. Channel emulation accuracy in terms of power angle delay profile, spatial correlation and beamforming power pattern are investigated. Simulation results demonstrate that with 16 OTA antennas uniformly distributed within $\left[-60^{\circ}, 60^{\circ}\right]$, the radio channels can be accurately reproduced for a $8 \times 8$ planar array BSs with half wavelength spacing at $3.5 \mathrm{GHz}$. For a $16 \times 16$ planar array BSs, though a similar

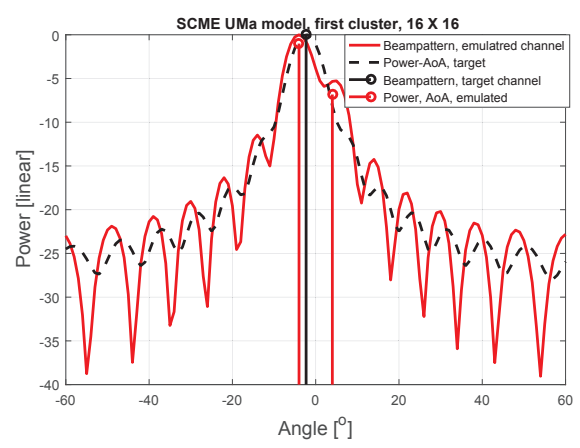

Figure 15. Beamforming power pattern of the first cluster in the target and emulated SCME UMa models with the $16 \times 16$ planar array. shape for beamforming power pattern can be still achieved, deviations exist in terms of spatial correlation, main beam and sidelobes for the beamforming power pattern. Therefore, more OTA antennas are needed for such large scale antenna systems. The sectorized MPAC setup is a cost-effective solution, compared to conventional conducted testing, since the number of required probe antennas might be significantly less than the number of antenna elements on the DUT. As demonstrated in the paper, both SCME channel models can be accurately reproduced within a test area of $5 \lambda \times 5 \lambda$ with 16 probes, where a 64 element planar array with half wavelength spacing can be supported. Note that 2D MPAC configuration is adopted for the sake of simplicity and selected 2D target channel models. For actual BS OTA testing, a 3D MPAC setup might be more suitable in the future. 3GPP 3D channel models and channel models above $6 \mathrm{GHz}$ emphasize the importance of modeling elevation domain. Further, planar arrays, not linear arrays, are typically adopted for massive MIMO BS systems.

\section{ACKNOWLEDGMENT}

This work has been partially supported by Huawei Technologies Sweden AB, Gothenburg, Sweden. Dr. Wei Fan would like to acknowledge the financial assistance from Danish council for independent research (grant number: DFF 611100525). The authors appreciate the valuable suggestions and comments from Dr. Gerhard Steinbock.

\section{REFERENCES}

[1] J. Vieira, S. Malkowsky, K. Nieman, Z. Miers, N. Kundargi, L. Liu, I. Wong, V. Owall, O. Edfors, and F. Tufvesson, "A flexible 100-antenna testbed for massive mimo," in Globecom Workshops (GC Wkshps), 2014, Dec 2014, pp. 287-293.

[2] T. Marzetta, "Massive MIMO: An Introduction," Bell Labs Technical Journal, vol. 20, pp. 11-22, 2015.

[3] Huawei, "Massive mimo takes prominent role in $5 \mathrm{~g}$ developments," 2015. [Online]. Available: http://www.rethinkresearch.biz/articles/massive-mimo-takesprominent-role-5g-developments/

[4] "Multi-Standard Radio (MSR) Base Station (BS) radio transmission and reception," 3GPP, TS 37.104 V12.6.0, December. 2014.

[5] "Test Plan for Wireless Device Overthe-Air Performance: Method of Measurement for Radiated RF Power and Receiver Performance," CTIA Certification, Tech. Rep. Version 3.5.2, September 2015.

[6] "Test Plan for 2x2 Downlink MIMO and Transmit Diversity Over-theAir Performance," CTIA Certification, Tech. Rep. Version 1.0, August 2015.

[7] "Active Antenna System (AAS) Base Station (BS) conformance testing; Part 2: radiated conformance testing ," CTIA Certification, Tech. Rep. Version V0.0.1, Release 13, February 2016.

[8] M. Gustafsson, T. Jämsä, A. Ahlström, M. Högberg, and B. Sihlbom, "Ota testing of base stations," The International Wireless Industry Consortium (IWPC), pp. 1-21, September 2015.

[9] M. Gustafsson, T. Jämsä, A. Ahlstrom, M. Högberg, and B. Sihlbom, "Over-the-Air (OTA) Testing of Base Stations ," COST IC1004, Tech. Rep. TD(15)13039, September 2015.

[10] P. Kyösti, W. Fan, G. F. Pedersen, and M. Latva-aho, "On dimensions of ota setups for massive mimo base stations radiated testing," IEEE Access, vol. 1, pp. 335-349, 2016.

[11] D. S. Baum, J. Hansen, J. Salo, G. Galdo, M. Milojevic, and P. Ky\{osti, "An interim channel model for beyond-3g systems: extending the $3 \mathrm{gpp}$ spatial channel model (scm)," in 2005 IEEE 61st Vehicular Technology Conference, vol. 5, May 2005, pp. 3132-3136 Vol. 5.

[12] "Verification of radiated multi-antenna reception performance of User Equipment," 3GPP, TR 37.977 V1.0.0, Sep. 2013.

[13] P. Kyösti, T. Jämsä, and J.-P. Nuutinen, "Channel modelling for multiprobe over-the-air mimo testing," International Journal of Antennas and Propagation, vol. 2012, 2012. 